\title{
Anaesthetic Management of Pulmonary Metastasectomy in a Patient Infected with Novel Corona Virus SARS-CoV-2
}

Mohammed J. Al-Naabi, ${ }^{1}$ "Madan M. Maddali, ${ }^{1}$ Karima R.S. Al Aliyani, ${ }^{2}$ Ahmed Al-Balushi ${ }^{3}$

\begin{abstract}
Prioritisation of individual patients for thoracic surgeries gained importance during the current COVID-19 pandemic to ensure optimal utilisation of resources. We report a 76-year-old-male patient who presented to a tertiary care hospital in Muscat, Oman, 2020, with bilateral pulmonary metastasis. The patient underwent an urgent pulmonary metastasectomy with successful anaesthesia management in an elderly patient despite him testing positive for COVID-19 twice via real-time reverse transcription-polymerase chain reaction. At the 3-month follow-up some pulmonary abnormalities persisted but the patient was recovering well. This report discusses the rationale to perform surgery in this case and highlights the precautions taken for reducing aerosol generation during the various stages of anaesthesia.
\end{abstract}

Keywords: COVID-19; Coronavirus Infections; One-Lung Ventilation; Personal Protective Equipment; Aerosols; Thoracic Surgical Procedures; Case Report; Oman.

\section{$\mathrm{T}$} HE EMERgEnCE OF THE SEVERE ACUte respiratory syndrome coronavirus 2 (SARSCoV-2), named the COVID-19 pandemic, has resulted in the prioritising of individual patients for thoracic surgeries in order to conserve resources. ${ }^{1}$ Droplets, contact and natural aerosols cause the spread of SARS-CoV-2. Thoracic anaesthesia for thoracic surgical procedures in patients infected with SARS-CoV-2 gain special attention as many inherent procedures involved are a major source of aerosol generation and may contribute to the speared of the virus. This case report describes the successful anaesthesia management of an elderly patient who tested positive twice for real-time reverse transcription-polymerase chain reaction (rRT-PCR) and needed a pulmonary metastasectomy as a semiemergency. This report highlights the rationale behind the decision for surgery and the precautions taken for reducing aerosol generation during the various stages of anaesthesia. To the best of the authors' knowledge, this is the first case report from Oman describing the practical aspects of anaesthetic management of a thoracic surgical procedure in a patient with COVID-19.

\section{Case Report}

A 76-year-old-male patient presented to a tertiary care hospital in Muscat, Oman, 2020, with bilateral pulmonary metastasis one year after having surgical excision of an adenocarcinoma of the sigmoid colon (pT4aN1b). The 18F-fluorocholine positron emission tomography/computed tomography imaging revealed an interval progression of the pulmonary metastases [Figure 1].

A left lung metastasectomy as a semi-emergency was planned as the metastatic nodule in the left lower lobe was rapidly increasing in size and was in close proximity to the pulmonary artery. At the time, the COVID-19 pandemic was at its peak and a preoperative rRT-PCR test as a screening investigation was done despite the patient being asymptomatic. Surgery was deferred as SARS-CoV-2 RNA was detected. After four weeks, the patient's rRT-PCR test was still positive. However, it was decided to proceed with the surgery as the cycle threshold $(\mathrm{Ct})$ value was 43 , above the cut off value of $34 .^{2,3}$ The cross-linked D-dimer assay XDP value was normal at $0.1 \mathrm{mg} / \mathrm{L}$ (normal range: $<0.5 \mathrm{mg} / \mathrm{L}$ ). A quantitative COVID-19 antibody test (anti-SARS-COV-2 S) done one day before surgery was negative $(<0.400 \mathrm{IU} / \mathrm{mL})$. The cardiopulmonary reserve assessed by a 6 -minute walk test was acceptable $(500 \mathrm{~m})$. The room air arterial blood gas analysis results were within the normal range $(\mathrm{pH}=7.4$, partial pressure of carbon dioxide $\left[\mathrm{pCO}_{2}\right]=40 \mathrm{mmHg}$, partial pressure of oxygen $\left[\mathrm{pO}_{2}\right]$ $=88 \mathrm{mmHg}$, oxygen saturation $=96 \%$, bicarbonate $\left[\mathrm{HCO}_{3}\right]=24 \mathrm{mmol} / \mathrm{L}$, sodium $[\mathrm{Na}+]=138 \mathrm{mmol} / \mathrm{L}$, potassium $[\mathrm{K}+]=4.2 \mathrm{mmol} / \mathrm{L}$, lactate $=1 \mathrm{mmol} / \mathrm{L}$ ); vital parameters were also normal (blood pressure = 140/88 $\mathrm{mmHg}$, heart rate $=78 / \mathrm{min}$ ). The patient could hold his breath for more than 10 seconds and he was using an incentive spirometer regularly. The chest X-ray displayed multiple nodular opacities related to 


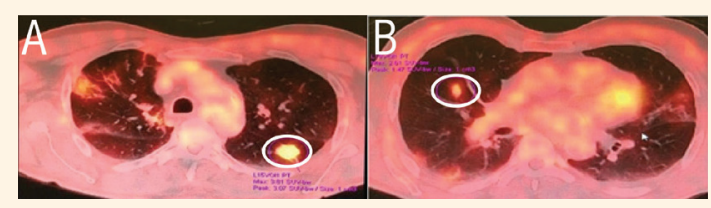

Figure 1: 18F-fluorocholine positron emission tomography/computed tomography imaging of the lungs of a 76-year-old-male patient showing metastatic nodules in the (A) left and (B) right lungs (circles).

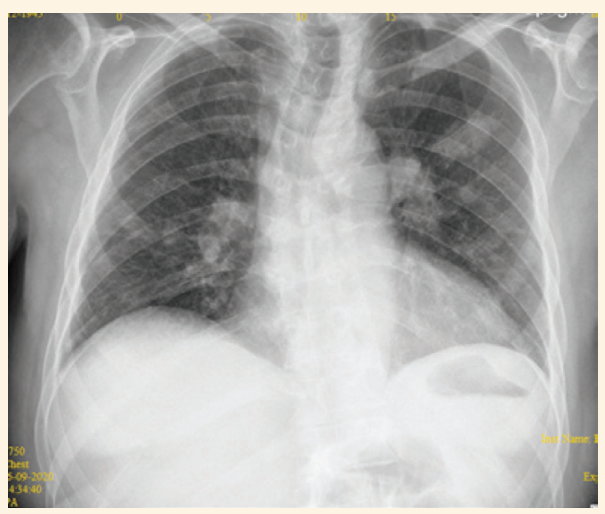

Figure 2: Chest radiograph of a 76-year-old-male patient in the anteroposterior view showing the lung fields with no signs of opacities or consolidation suggestive of COVID-19 infection of the lungs.

the underlying malignancy. There were no ground glass opacities or evidence of consolidation suggestive of a COVID-19 involvement of the lungs [Figure 2].

After obtaining informed consent for the surgical procedure, the patient was shifted to the operation room. All personnel involved, donned personal protective equipment (PPE). The positive-pressure system and air conditioning in the operating room was turned off. After preoxygenation with oxygen through a tight-fitting face mask for three minutes, general anaesthesia was administered under standard American Society of Anesthesiologists monitoring protocol. The patient's trachea was intubated with a $37 \mathrm{Fr}$ double lumen tube (DLT) with the help of a video laryngoscope. The DLT was positioned and the placement was confirmed by fibreoptic bronchoscopy (FOB) with the patient being apnoeic. The tracheal and bronchial cuffs of the DLT were inflated immediately to avoid leakage of aerosol. High-efficiency particulate air (HEPA) filters were applied between the disposable anaesthesia face mask and the breathing circuit, on the inspiratory and expiratory limbs of the breathing circuit, between the DLT catheter mount and the breathing circuit and on the bronchial lumen of the DLT that would be in communication with the operative non-dependent lung adding up to a total of five HEPA filters.

A pressure-controlled mode of mechanical ventilation was initiated with a fraction of inspired oxygen $\left(\mathrm{FiO}_{2}\right)$ of 0.7 and a positive end expiratory pressure of $5 \mathrm{cmH}_{2} \mathrm{O}$. Pressure limit was adjusted to achieve $6 \mathrm{~mL} / \mathrm{kg} / \mathrm{min}$ tidal volume monitoring the end tidal carbon dioxide. General anaesthesia was maintained with infusions of dexmedetomidine (0.6 $\mathrm{mcg} / \mathrm{kg} / \mathrm{hr}$ ), cisatracurium, fentanyl and sevoflurane (0.6 to $1 \mathrm{MAC}$ ). One lung ventilation was achieved without any episode of hypoxaemia. Left parenchymal sparing metastasectomy with anatomical

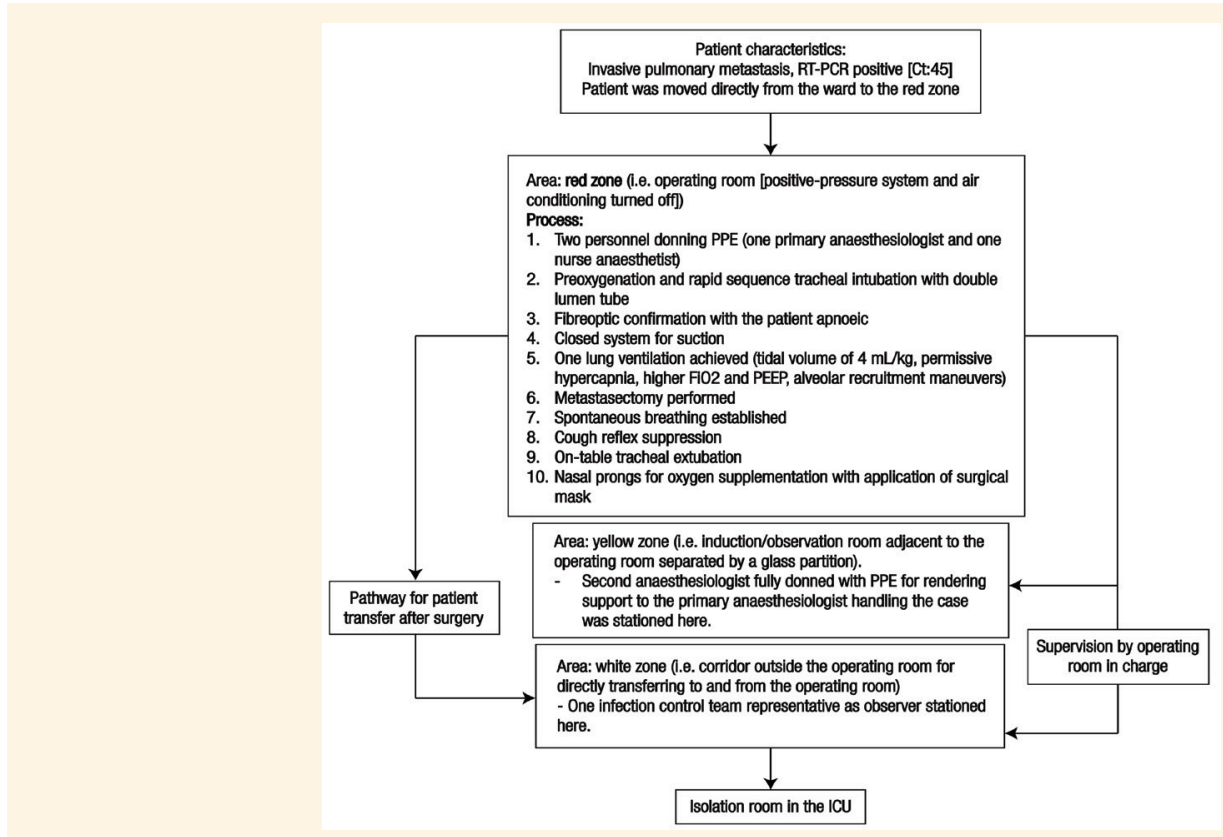

Figure 3: Flowchart showing the stages of patient management and the zones in the operating room. $R T-P C R=$ reverse transcription-polymerase chain reaction; $C t=$ cycle threshold; $P P E=$ personal protective equipment; FiO2 = fraction of inspired oxygen; PEEP = positive end expiratory pressure; $I C U=$ intensive care unit. 
segmentectomy was performed and six metastatic masses were removed. On completion of the procedure, aspiration of secretions was performed via a closed system from the non-dependent of the lung, followed by an alveolar recruitment maneuver of the collapsed lung and initiation of both lung ventilation. The wound was closed in layers; the surgery took approximately 95 minutes.

One intercostal drain was inserted that was connected to an Argyle ${ }^{\mathrm{TM}}$ Thora-Seal $^{\mathrm{TM}}$ III chest drain system (Cardinal Health, Dublin, Ireland). Synchronised intermittent mandatory ventilation mode was initiated on return of spontaneous breathing. This was changed to pressure support mode $\left(10 \mathrm{cmH}_{2} \mathrm{O}\right)$ and the neuromuscular blockade was reversed. The cough reflex was inhibited with dexmedetomidine infusion and a bolus of lidocaine injection $(1 \mathrm{mg} / \mathrm{kg})$. All anaesthetic agents were stopped once surgical dressing was completed. $\mathrm{FiO}_{2}$ of $100 \%$ was administered for two minutes and the patient's trachea was extubated. Oxygen was administered through nasal prongs and a surgical face mask was applied to the patient's face. An arterial blood gas analysis was done $\left(\mathrm{pH}=7.39, \mathrm{PaCO}_{2}=37 \mathrm{mmHg}\right.$, $\mathrm{PO}_{2}=90 \mathrm{mmHg}$, oxygen saturation $=99 \%, \mathrm{HCO}_{3}=22$ $\mathrm{mmol} / \mathrm{L}, \mathrm{Na}^{+}=136 \mathrm{mmol} / \mathrm{L}, \mathrm{K}^{+}=3.2 \mathrm{mmol} / \mathrm{L}$, lactate $=1.9 \mathrm{mmol} / \mathrm{L})$. After confirmation of uncompromised spontaneous breathing, the patient was transferred to the intensive care unit (ICU). The intercostal drain was removed after 48 hours postoperatively and the patient was discharged home on the seventh day after surgery. The patient's flow diagram shows the stages of management of the patient as well as the zones in the operating room [Figure 3].

A computed tomography (CT) scan done at the 3-month follow-up reported diffuse bronchial wall thickening with patent central tracheobronchial tree. There was mild para-septal emphysema with bi-apical scarring. The left apical region displayed cicatrisation and atelectasis. Institutional ethical committee approval [SRC\#CR22/2020] and informed consent from the patient were obtained for publishing this report.

\section{Discussion}

This report describes the successful anaesthetic management of an elderly patient who had a positive rRT-PCR test and needed urgent pulmonary metastasectomy. The recommendation for timing the surgery in patients with SARS-CoV-2 infection has changed recently. At the time the current patient was operated on, the recommendation was that once SARS-CoV-2 infection was diagnosed, surgery was to be postponed for at least 7-14 days from the end of symptoms and should be followed by a negative test result. ${ }^{4,5}$ Recent evidence suggests that surgery should be delayed by seven weeks. ${ }^{6}$

However, a positive rRT-PCR assay result alone may not be as important as the $\mathrm{Ct}$ value. The $\mathrm{Ct}$ threshold value is the number of amplification cycles needed to yield a positive fluorescent signal in a rRTPCR test and is a surrogate marker for the viral load. Ct levels are inversely proportional to the amount of target nucleic acid in the sample. At the time of surgery for the current patient, data suggested 34 cycles as the cut-off value for determining on SARS$\mathrm{CoV}-2$ infectivity. ${ }^{2}$ A patient may not be infectious if Ct values are $\geq 35$. $^{3}$

Viral droplets are the principal cause of the spread of COVID-19 infection. ${ }^{4}$ Many anaesthetic procedures result in aerosol generation of viral particles that may pose a risk for the medical team taking care of COVID-19 positive patients. Stress on minimising aerosol generation during the various stages of anaesthesia is the primary difference in anaesthetising a COVID-19 negative patient for thoracic surgery compared to those who test positive for COVID-19. The European Association of Cardiothoracic Anaesthesiology Thoracic Subspecialty Committee published updated recommendations for the management of thoracic anaesthesia patients with suspected or confirmed COVID-19 infection. ${ }^{4}$ The recommendations emphasise achieving successful airway management without compromising the health of the treating team. Guidelines are also available for triaging patients with thoracic malignancies based on the trajectory and the impact of COVID-19 cases on each institution. ${ }^{1}$ Notwithstanding a rapidly escalating COVID-19 trajectory with many hospital admissions and resources constraint, the current patient underwent early surgery.

During preoperative evaluation of suspected COVID-19 patients, rRT-PCR testing and when its unavailable, chest $\mathrm{CT}$ needs to be done. ${ }^{5}$ There might be COVID-19-related blood test anomalies such as elevated values of C-reactive protein, erythrocyte sedimentation rate and D-dimer levels. ${ }^{5}$ COVID-19 patients may exhibit chest radiography abnormalities as well. ${ }^{5}$ The current patient tested positive for COVID-19 on rRT-PCR on two occasions with a $\mathrm{Ct}$ of $>35$ the second time. As the metastatic lesions were rapidly increasing in size with the possibility of infiltration into pulmonary vasculature, the decision to operate was made.

Tracheal intubation is to be performed electively using rapid sequence induction with complete neuromuscular paralysis after preoxygenation in 
an 'isolated' negative pressure room with $>12$ air changes/minute. ${ }^{4}$ In the current patient, as a negative pressure operating room was not available, the tracheal intubation was done in an operating room after switching off the positive pressure with the doors closed.

The operating room area was separated into red, white and yellow zones. ${ }^{4}$ In the red zone (i.e. the operating room), there were two staff members donned with full PPE during the tracheal intubation that was performed by a senior anaesthesiologist with the assistance of a nurse. The yellow zone was the induction room that was attached to the operating room and separated by a glass partition where an anaesthesiologist with full PPE was available in case assistance was required as suggested by Şentürk et al. ${ }^{4}$ An observer from the institutional infection control team was present in the white zone that was outside the dedicated operating room. The PPE donned by the team comprised of hair covers, two N95 face masks each, face shield, long sleeve fluid-resistant gown, double gloves and overshoes as prescribed. ${ }^{4}$ According to the European Guidelines, lung separation with a bronchial blocker maybe the preferred mode in COVID-19 patients undergoing thoracic surgery. ${ }^{4}$ This technique mandates the use of an FOB guidance.

Simultaneously, many other international societies recommend consideration of DLTs in lieu of bronchial blockers for patients who do not have a known or suspected difficult airway with the placement of an antiviral filter on the open non-ventilated lumen of the DLT., ${ }^{7,8}$ A DLT may be positioned without the help of FOB guidance based on auscultation and may be a better choice of lung isolation in COVID-19 postive patients. In the current patient, an initial attempt was made to place a DLT without the help of FOB guidance. As this initial attempt failed, it necessitated the need for FOB for accurate placement of the DLT during the second attempt. This was done maintaining the patient apnoeic status. The management guidelines suggested by Şentürk et al. for the conduct of one lung ventilation in patients with COVID-19 were used in the current case. ${ }^{4}$ The placement of HEPA filters in the anaesthesia circuit, management of ventilation parameters, alveolar recruitment techniques prior to resumption of both lung ventilation after achieving one lung ventilation, use of closed system for aspiration of secretions, cough suppression at the time of tracheal extubation were in accordance with these guidelines. ${ }^{4}$ Nasal prongs for oxygen supplementation were inserted in the patient with the application of a surgical face mask over the prongs. Observation of the patient during the immediate post-extubation period was done in the operating room and once the anaesthesiologist was convinced of the cardio-pulmonary stability of the patient, the patient was shifted to an isolation room in the ICU for further management.

Attention was paid towards the possibility of virus transmission via the chest drainage unit. Following the metastasectomy, no air leak was detected during the process of recruitment and expansion of the lung that was operated on. The intercostal drain was connected to a chest drain unit that was a closed system which had three chambers: a collection chamber for pleural fluid/ chest drain output, a water-seal chamber that acted as a one-way valve and a suction control chamber set to negative pressure of $5 \mathrm{cmH}_{2} \mathrm{O}$. The outlet port of the third chamber was connected to the wall-mounted suction to scavenge the exhaust from any ongoing air leak or air displaced by pleural drainage in the operating room and in the ICU. During transport to the ICU, the outlet port was kept sealed. In order to reduce the possibility of disease transmission, it has been suggested to attach a HEPA filter to the port of the drainage system that is open to the environment. ${ }^{9}$

Based on prior experience of management of patients during the Middle East Respiratory Syndrome-CoV outbreak and the available literature, guidelines for anaesthesia management of thoracic surgery in patients with suspected/confirmed COVID-19 were recently published by the Saudi Anesthesia Society. ${ }^{10}$ These guidelines address the general regulations, organisation, preparations and lung isolation/separation in different types of patients during a COVID-19 outbreak. ${ }^{10}$ Many of these were adopted in the current patient's management.

\section{Conclusion}

Case selection for thoracic surgeries during the current COVID-19 pandemic must be based on multidisciplinary discussion; the timing of surgery may be dictated by the patients underlying pathology. The perioperative anaesthetic management poses an important challenge as many events that are necessary for the conduct of anaesthesia in these patients are a major source of aerosol generation and may contribute to the spread of the infection. Therefore, the primary objective of the conduct of thoracic anaesthesia in COVID-19 positive patients is to minimise aerosol generation at all stages.

\section{AUTHORS' CONTRIBUTION}

MMM conceptualised the work and was involved in acquisition of the images and drafted the manuscript. MJN, MMM, KRSA and AB were involved in the interpretation of the data. MJN was responsible 
for the provision of the anaesthesia details. $A B$ was responsible for the provision of the operation data. KRSA collected the data. All authors approved the final version of the manuscript.

\section{References}

1 Thoracic Surgery Outcomes Research Network, Inc, AntonoffM, Backhus L, Boffa DJ, Broderick SR, Brown LM, et al. COVID-19 Guidance for Triage of Operations for Thoracic Malignancies: A consensus statement from thoracic surgery outcomes research network. Ann Thorac Surg 2020; 110:692-6. https://doi.org/10.10 16/j.jtcvs.2020.03.061.

2. Engelmann I, Alidjinou EK, Ogiez J, Pagneux Q, Miloudi S, Benhalima I, et al. Preanalytical Issues and Cycle Threshold Values in SARS-CoV-2 Real-Time RT-PCR Testing: Should test results include these? ACS Omega 2021; 6:6528-36. https://doi. org/10.1021/acsomega.1c00166.

3. Zacharioudakis IM, Prasad PJ, Zervou FN, Basu A, Inglima K, Weisenberg SA, et al. Association of SARS-CoV-2 Genomic Load with Outcomes in Patients with COVID-19. Ann Am Thorac Soc 2021; 18:900-3. https://doi.org/10.1513/AnnalsAT S.202008-931RL.

4. Sentürk M, El Tahan MR, Szegedi LL, Marczin N, Karzai W, Shelley B, at al. Thoracic Anesthesia of Patients With Suspected or Confirmed 2019 Novel Coronavirus Infection: Preliminary recommendations for airway management by the European Association of Cardiothoracic Anaesthesiology Thoracic Subspecialty Committee. J Cardiothorac Vasc Anesth 2020; 34:2315-27. https://doi.org/10.1053/j.jvca.2020.03.059.
5. Fiorelli S, Menna C, Piccioni F, Ibrahim M, Rendina EA, Rocco M, et al. The Cutting Edge of Thoracic Anesthesia During the Coronavirus Disease 2019 (COVID-19) Outbreak. J Cardiothorac Vasc Anesth 2020; 34:3203-10. https://doi.org/10.1053/j. jvca.2020.05.042.

6. COVIDSurg Collaborative; GlobalSurg Collaborative. Timing of surgery following SARS-CoV-2 infection: An international prospective cohort study. Anaesthesia 2021; 76:748-58. https:// doi.org/10.1111/anae.15458

7. Shaylor R, Verenkin V, Matot I. Anesthesia for Patients Undergoing Anesthesia for Elective Thoracic Surgery During the COVID-19 Pandemic: A consensus statement from the Israeli Society of Anesthesiologists. J Cardiothorac Vasc Anesth 2020; 34:3211-17. https://doi.org/10.1053/j.jvca.2020.07.049.

8. Thornton M, Reid D, Shelley B, Steven M. Management of the airway and lung isolation for thoracic surgery during the COVID-19 pandemic: Recommendations for clinical practice endorsed by the Association for Cardiothoracic Anaesthesia and Critical Care and the Society for Cardiothoracic Surgery in Great Britain and Ireland. Anaesthesia 2020; 75:1509-16. https://doi.org/10.1111/anae.15112.

9. Ghoniem A, Abdellateef A, Osman AI, Elsayed HH, Elkhayat H, Adel W. A tentative guide for thoracic surgeons during COVID19 pandemic. Cardiothorac Surg 2020; 28:16. https://doi.org/1 0.1186/s43057-020-00026-z.

10. Eldawlatly A, El Tahan MR, Abdulmomen A, Kattan M, Ahmad AE. Anesthesia management of thoracic surgery in a patient with suspected/confirmed COVID-19: Interim Saudi Anesthesia Society guidelines. Saudi J Anaesth 2020; 14:383-6. https://doi. org/10.4103/sja.SJA_252_20. 